\title{
Robust and Undetectable Steganographic Timing Channels for i.i.d. Traffic
}

\author{
Tracy(Yali) Liu and Dipak Ghosal \\ Network Labs \\ University of California, Davis, USA
}

Frederik Armknecht, Ahmad-Reza Sadeghi and Steffen Schulz System Security Lab

Ruhr-Universität Bochum, Germany
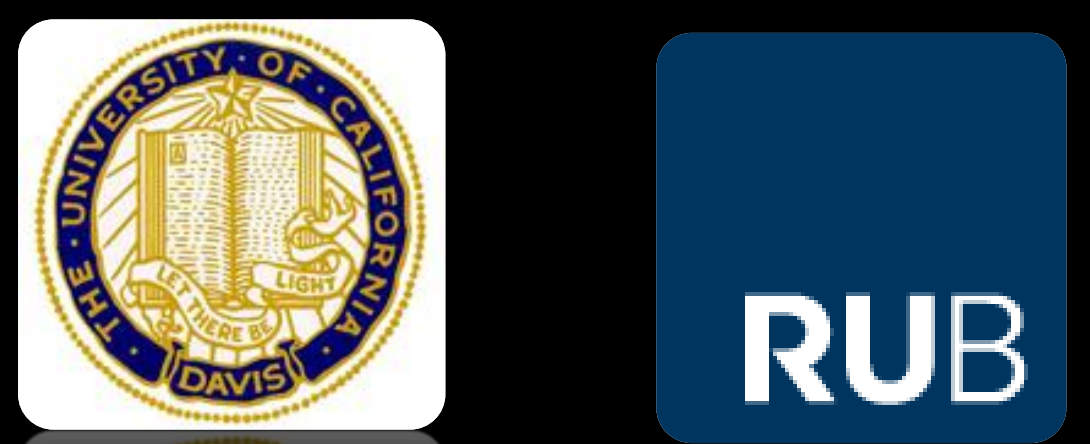

Stefan Katzenbeisser Security Engineering Group

TU Darmstadt, Germany 


\section{Main Motivation:}

Steganographic Communication over Network Traffic

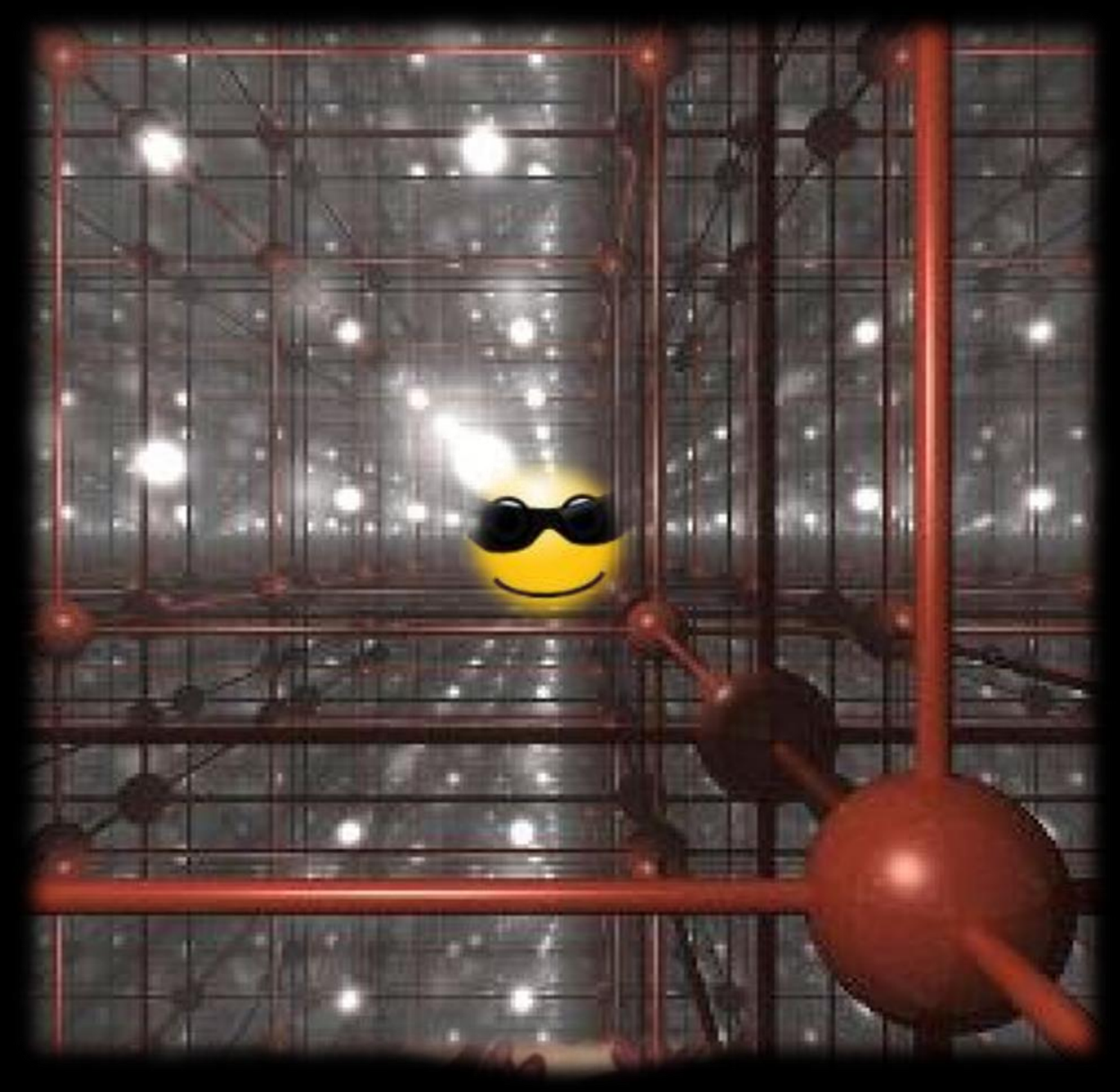




\section{Steganographic Channels}

\section{$\diamond$ Common types}

Storage channels - communicate by modifying a stored object

$\diamond$ Timing channels - transmit information by affecting the relative timing of events

$\diamond$ Requirements

$\checkmark$ Robustness - resilience to noise

$\diamond$ Security - undetectable by the adversary 


\section{Our Focus}

$\diamond$ Timing channels based on inter-packet delays , i.e., the sending delays between successive packets.

$\diamond$ More concretely, independent and identically distributed (i.i.d.)

Why i.i.d. traffic

$\diamond$ Extensively used in existing network analysis

$\diamond$ Essential element in many advanced traffic models 


\section{Existing solutions.....}




\section{Existing Solutions and Problems}

Common steganographic timing channels

$\checkmark$ On and off

"small-delays" and "large-delays"

$\checkmark$ Perturb the inter-packet delays through small variations

Encoding scheme design to maximize the channel capacity i.i.d. solution

$\diamond$ Counter measures to disrupt and/or detect steganographic traffic

e.g., timing jammers, statistical tests

$\diamond$ Problems

Security is only guaranteed under certain conditions

$\checkmark$ Robustness is not sufficient against noisy channels or a malicious jammer 


\section{Our Contribution}

$\diamond$ A novel steganographic timing channel for any legitimate traffic whose inter-packet delays are i.i.d. following an arbitrary distribution

$\checkmark$ Undetectable against any (efficiently computable) statistical test

$\diamond$ Robust against disruptions (caused by active adversaries and/or network noise)

$\diamond$ Tunable encoding parameters allow to trade-off

$\diamond$ Robustness

$\diamond$ Transmission rate

$\diamond$ Validation on real telnet traffic under different network conditions 


\section{Steganographic Channel in Telnet Traffic}

-Telnet traffic: i.i.d. inter-packet delays

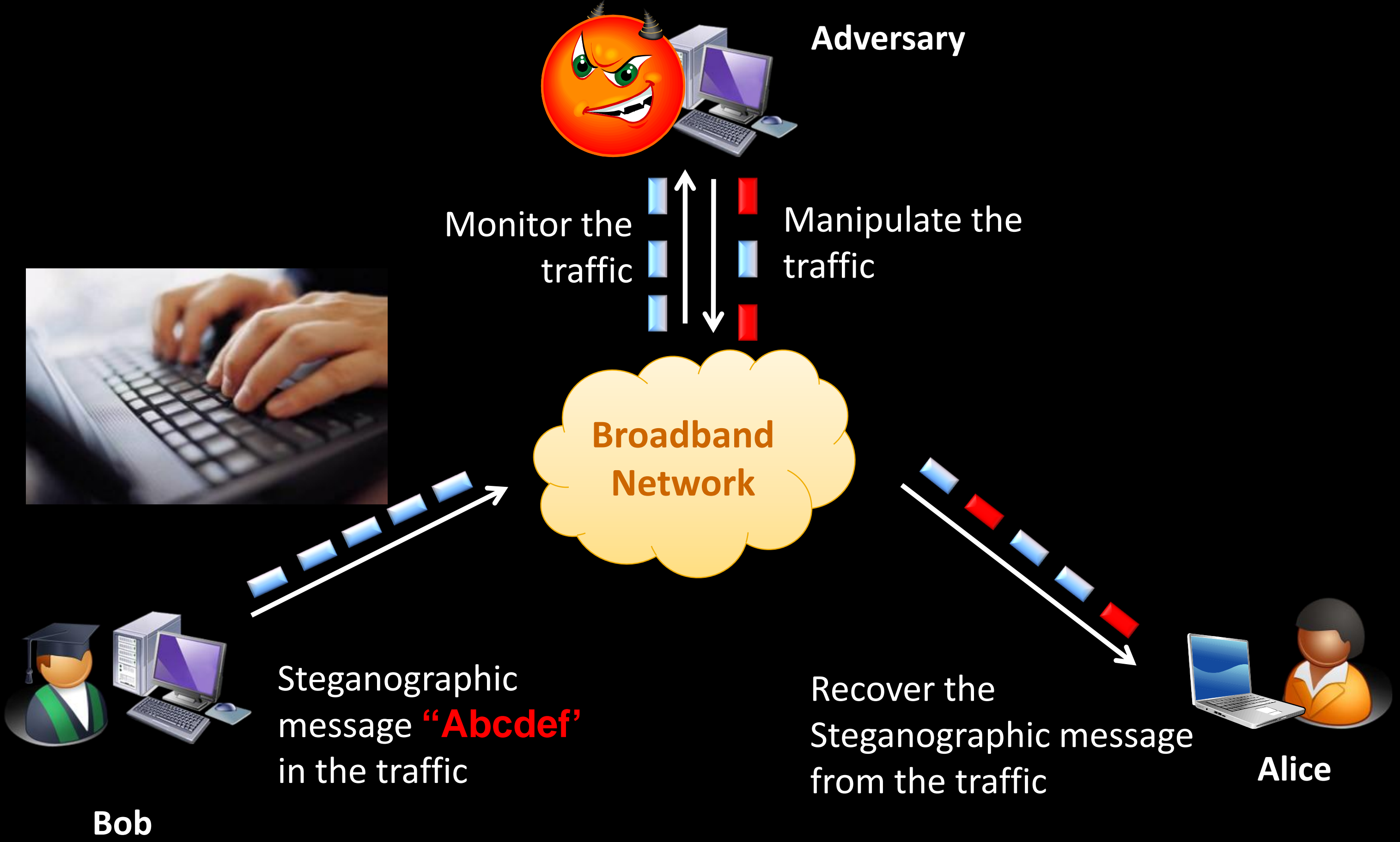


And Our Solution.... 


\section{Design Objectives \& Requirements}

$\checkmark$ Undetectability

$\checkmark$ Indistinguishability: adversary cannot indistinguish between the legitimate and steganographic traffic

$\diamond$ Robustness

$\diamond$ Resistance to noise (malicious or non-malicious)

$\diamond$ Decoding error probability: Bit Error Rate (BER) $P_{e}$

$\checkmark$ Robustness gain: time to increase SNR $\gamma$

$\diamond P_{e}$ is inverse function of SNR 


\section{System Overview}

\section{Steganographic sender}

Steganographic message $\left\{b_{1}, b_{2}, b_{3} \cdots\right\}$

Legitimate packet

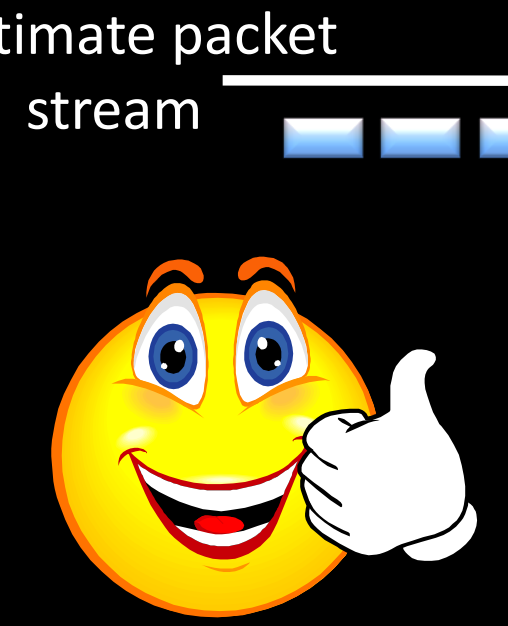

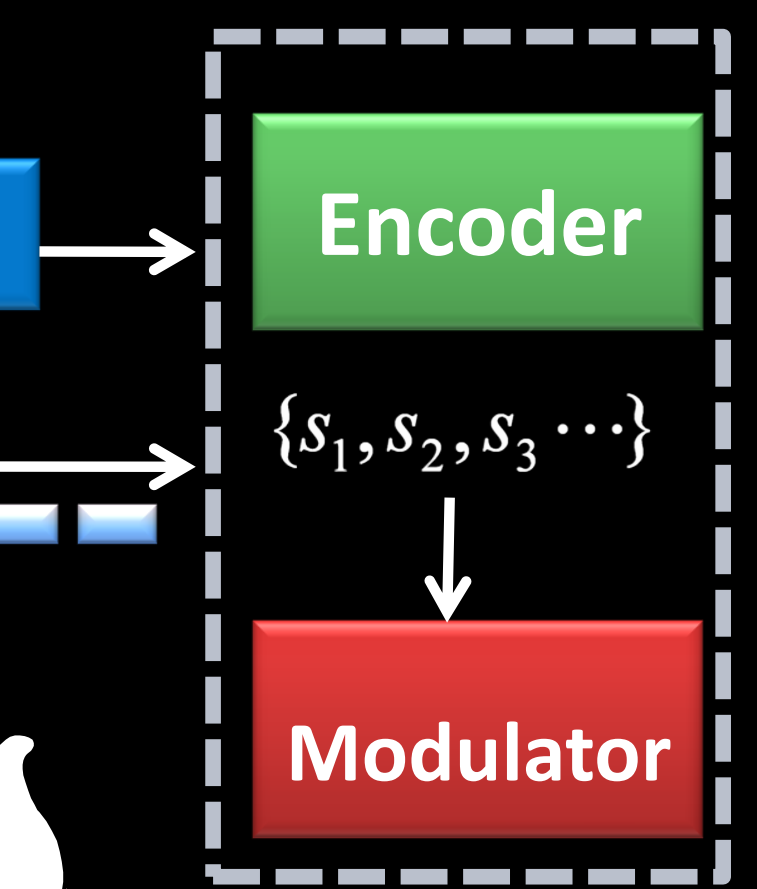

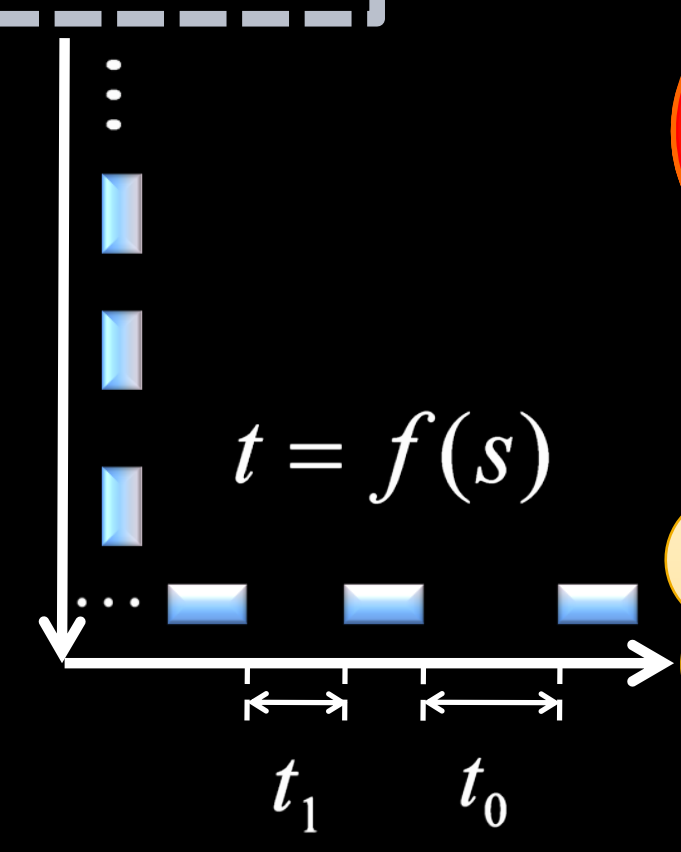

\section{Adversary}

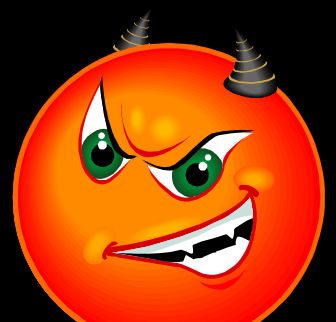

- Monitor

- Manipulate

- Detection
Steganographic receiver

$$
\left\{\hat{b}_{1}, \hat{b}_{2}, \hat{b}_{3} \cdots\right\}
$$

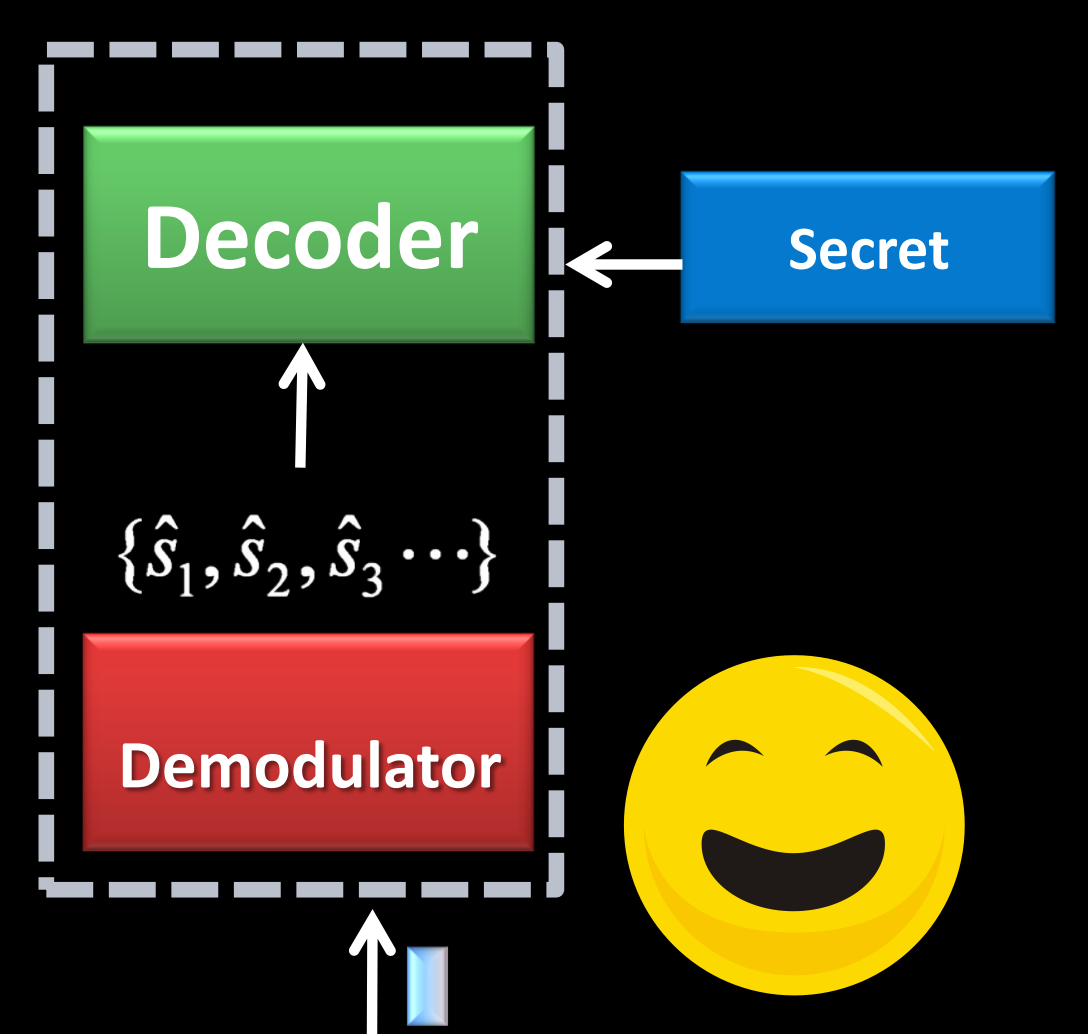




\section{Encoding with Spreading Codes}

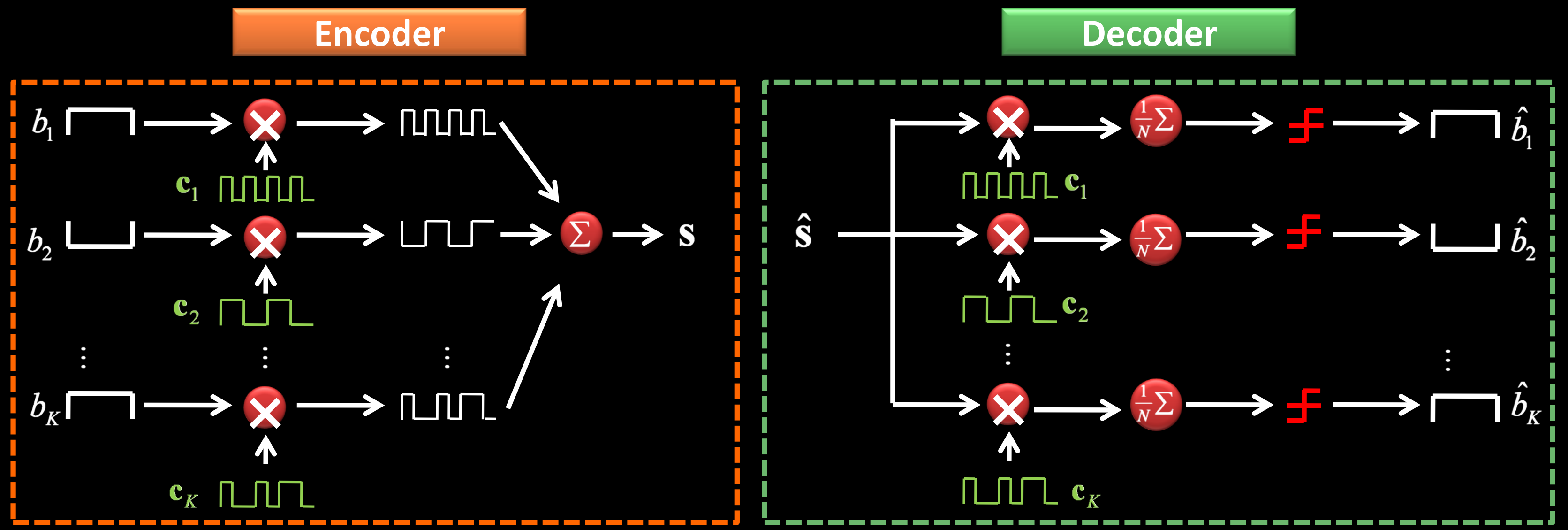

$\checkmark$ Uses unique spreading codes to spread the baseband data before transmission

$\checkmark$ Low bit error rate (BER) - spreading gain $N$

$\diamond$ Noise power decreases by $N$

$\diamond$ High transmission rate - orthogonal codes

$$
\diamond \mathbf{s}=\sum_{k=1}^{K} b_{k} \cdot \mathbf{c}_{k} \quad\left\langle\mathbf{c}_{i}, \mathbf{c}_{j}\right\rangle=\left\{\begin{array}{ccl}
1 & \text { if } i=j & K: \text { total number of channel } \\
0 & \text { otherwise } & R_{t}=K / N: \text { transmission rate }
\end{array}\right.
$$




\section{Modulation to Address Statistical Detection}

$\diamond$ Function

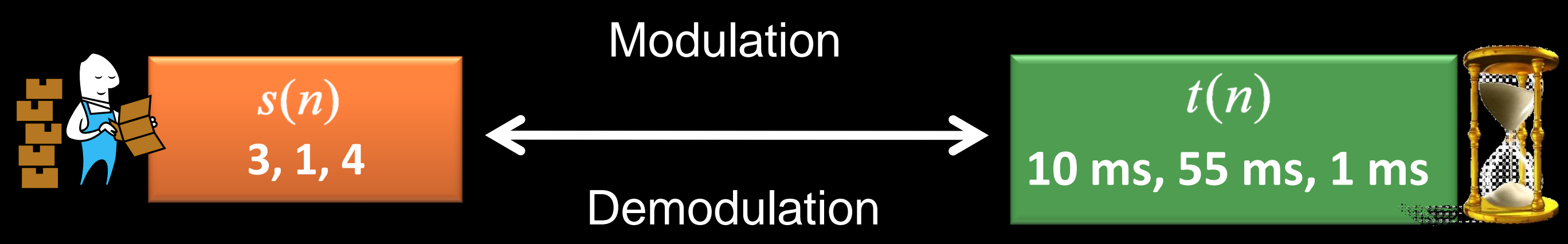

$\diamond$ Priori knowledge

$\diamond$ Characteristics of the legitimate network traffic

$\diamond$ Requirements

$\diamond$ Invertible mapping

$\diamond$ Evade any statistical tests 


\section{Undetectable Modulation (1)}

$\diamond$ Inverse function based modulation scheme

$\diamond u(n)=F_{s}\left(l_{m-1}\right)+\left(F_{s}\left(l_{m}\right)-F_{s}\left(l_{m-1}\right)\right) \cdot v(n)$

$\diamond d(n)=F_{\widetilde{d}}^{-1}(u(n))$

$\diamond F_{s}(\cdot)$ CDF of code symbol $\mathrm{s}(\mathrm{n})$

$\diamond F_{\tilde{d}}(\cdot)$ CDF of legitimate traffic
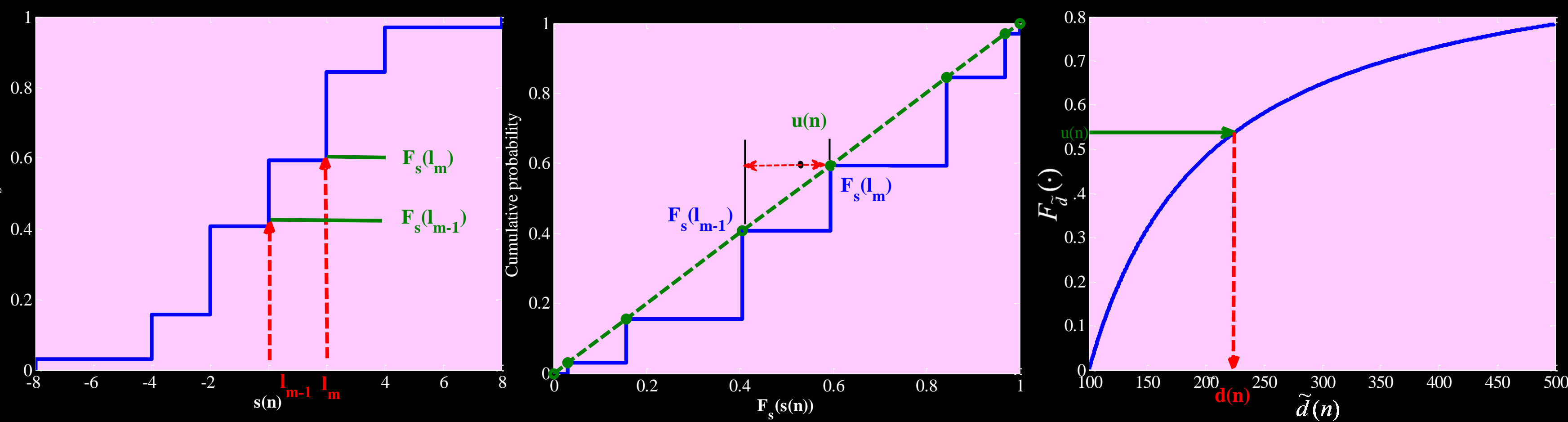


\section{Undetectable Demodulation (2)}

$\diamond$ Additive noise during transmission

$\diamond \hat{d}(n)=d(n)+x(n)$

$\checkmark$ Inverse function based demodulation scheme

$\diamond \hat{u}(n)=F_{\widetilde{d}}(\hat{d}(n))$

$\diamond \hat{s}(n)=l_{m} \quad$ if $\hat{u}(n)=\in\left(F_{s}\left(l_{m-1}\right), F_{s}\left(l_{m}\right)\right]$

$\diamond \hat{b}_{k}=\frac{1}{N}\left(\hat{\mathbf{s}}, \mathbf{c}_{k}\right)=b_{k}+\frac{1}{N}\left\langle\mathbf{x}, \mathbf{c}_{k}\right\rangle$



\section{Determining Model Parameters}

$\diamond$ Modulation - compression

$\diamond$ Robustness gain - effective processing gain

$\diamond$ The SNR after performing the encoding and modulation process to the one without encoding and modulation scheme.

$$
\gamma=N \frac{1}{M} \sum_{i=1}^{M} \frac{\left(F_{s}\left(l_{i}\right)-F_{s}\left(l_{i-1}\right)\right)^{2}}{\left(\frac{1}{2}\right)^{2}}=\frac{4 N}{K+1}\left(\frac{1}{2}\right)^{2 K} \sum_{j=0}^{K}\left(\begin{array}{c}
K \\
j
\end{array}\right)^{2}
$$
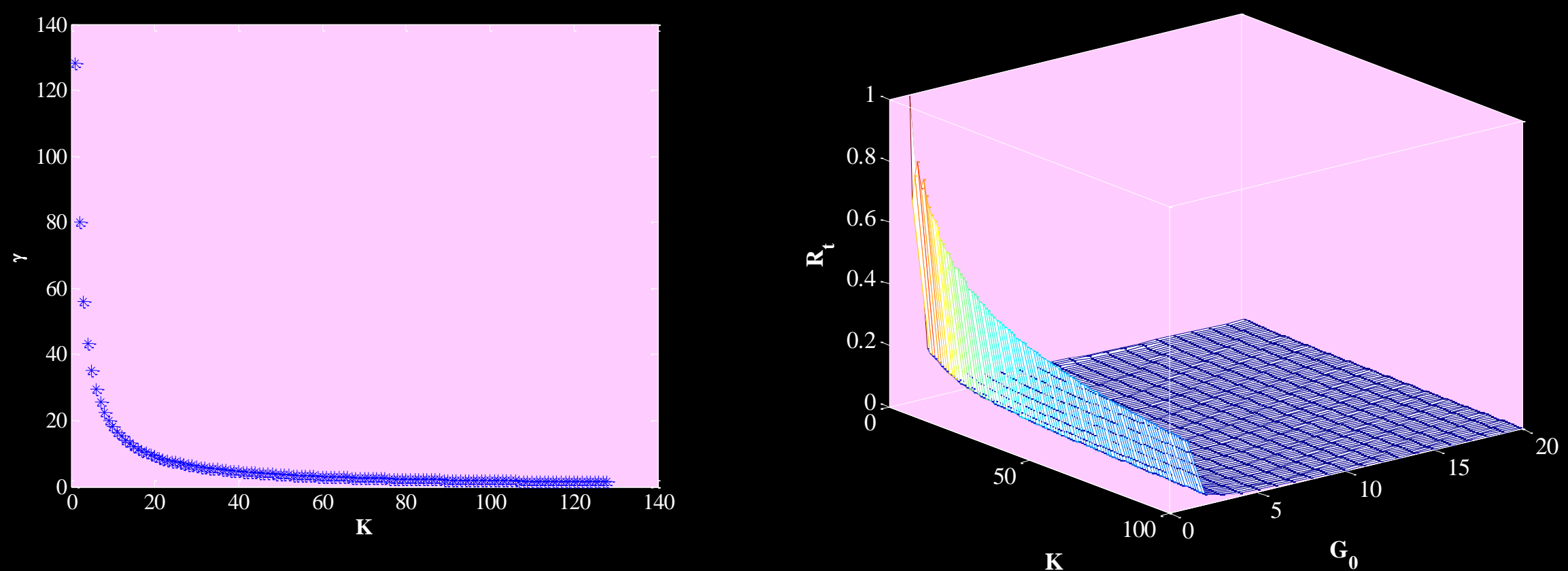


\section{Algorithm Summary}

Execution

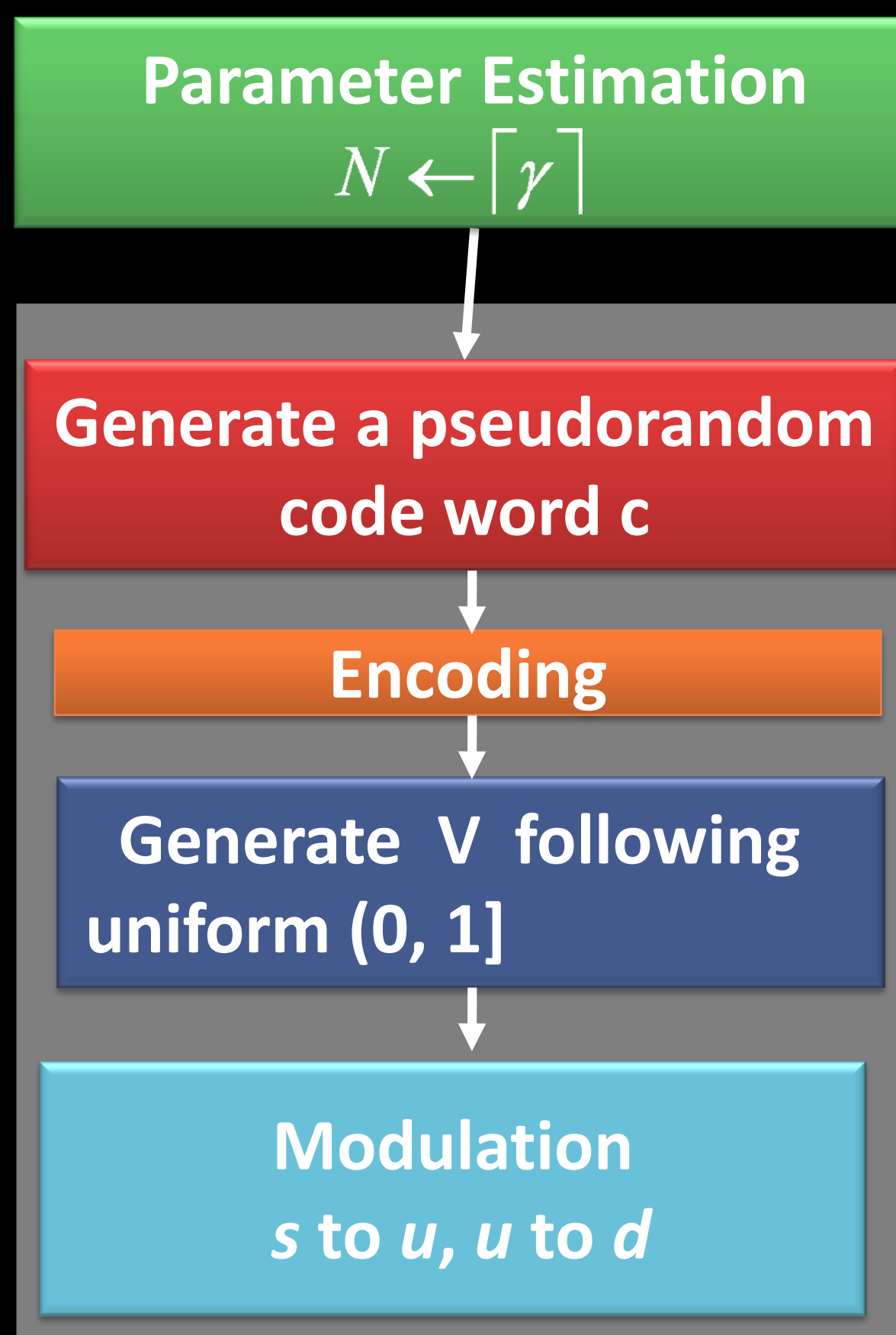

Input to the system

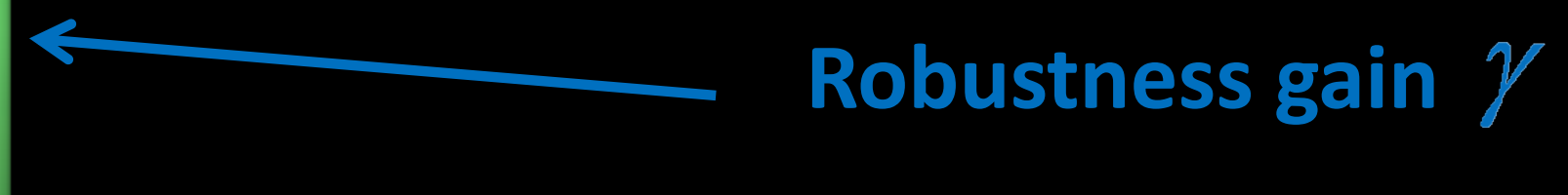

Key

Legitimate inter-packet delay distribution $F_{\widetilde{d}}(\cdot)$

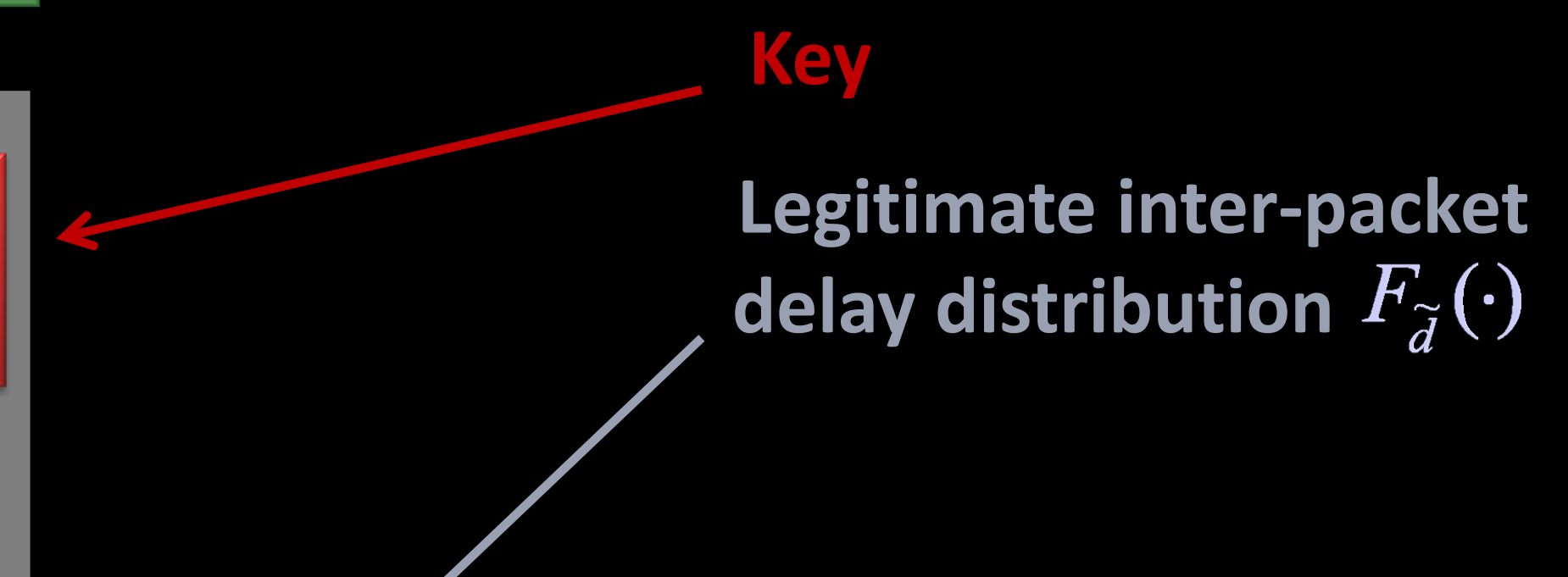

Output

Covert inter-packet delays $d(1), d(2) \ldots$ 


\section{Experimental Setup}

\section{Simulation of the legitimate traffic}

$\diamond$ Network client sends packets in exact same inter-packet delays as desired traffic

$\diamond$ Content of packets is a counter to identify packet loss, dupes and order of arrival

\section{Physical setup}

$\diamond$ WAN: Two Linux servers at RUB and UC Davis

$\diamond$ LAN: Two Linux servers at UC Davis

$\diamond$ Active adversary

$\diamond$ A network sniffer at the receiver

$\diamond$ Injects noise at the sender

Real traffic traces from online archive dataset: MAWI working group traffic archive 


\section{Implementation Architecture}

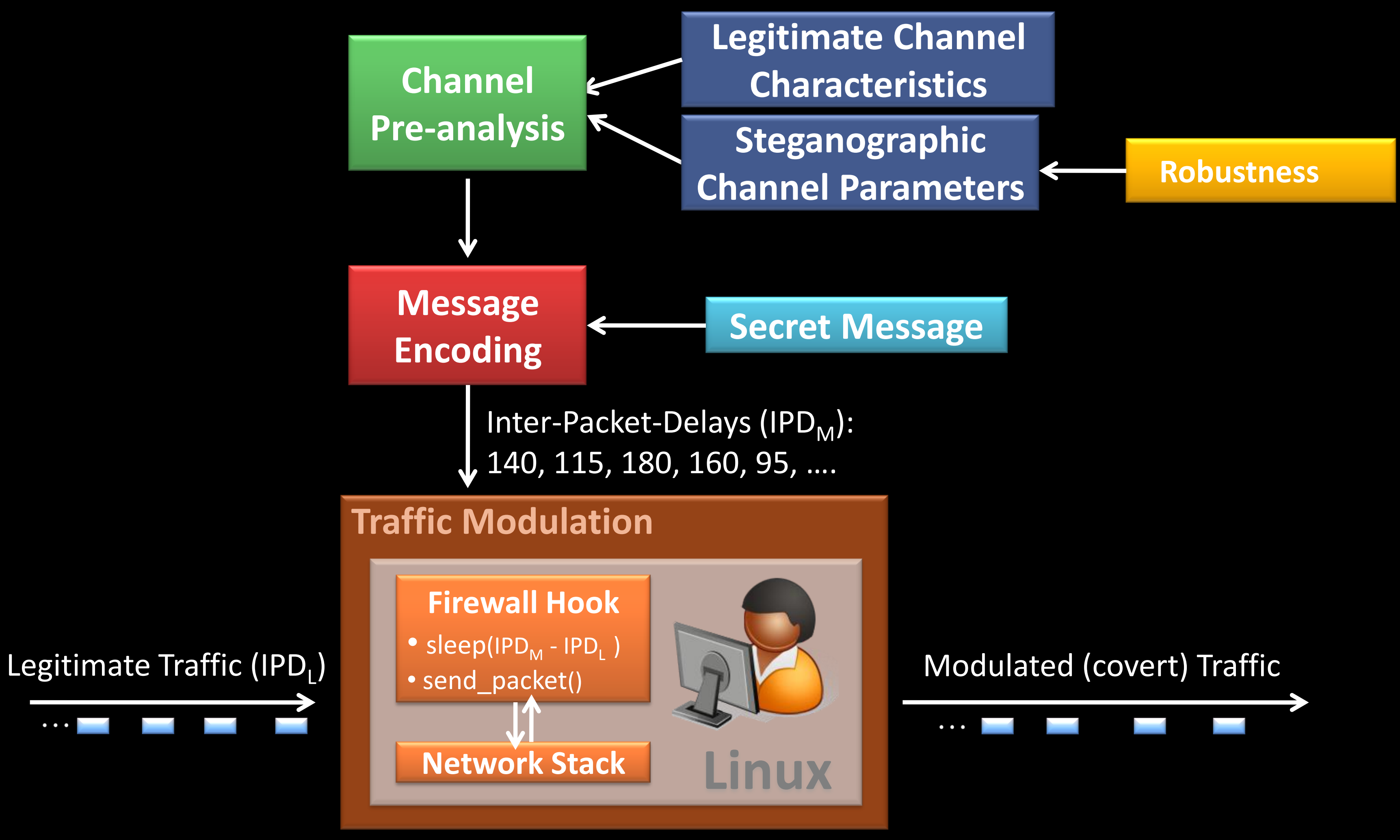




\section{Undetectability Visualization}

Telnet $F(\widetilde{d})=1-\left(\frac{\alpha}{\widetilde{d}}\right)^{\beta}$ scale parameter $\alpha=49 \mathrm{~ms}$, shift parameter $\beta=0.93$

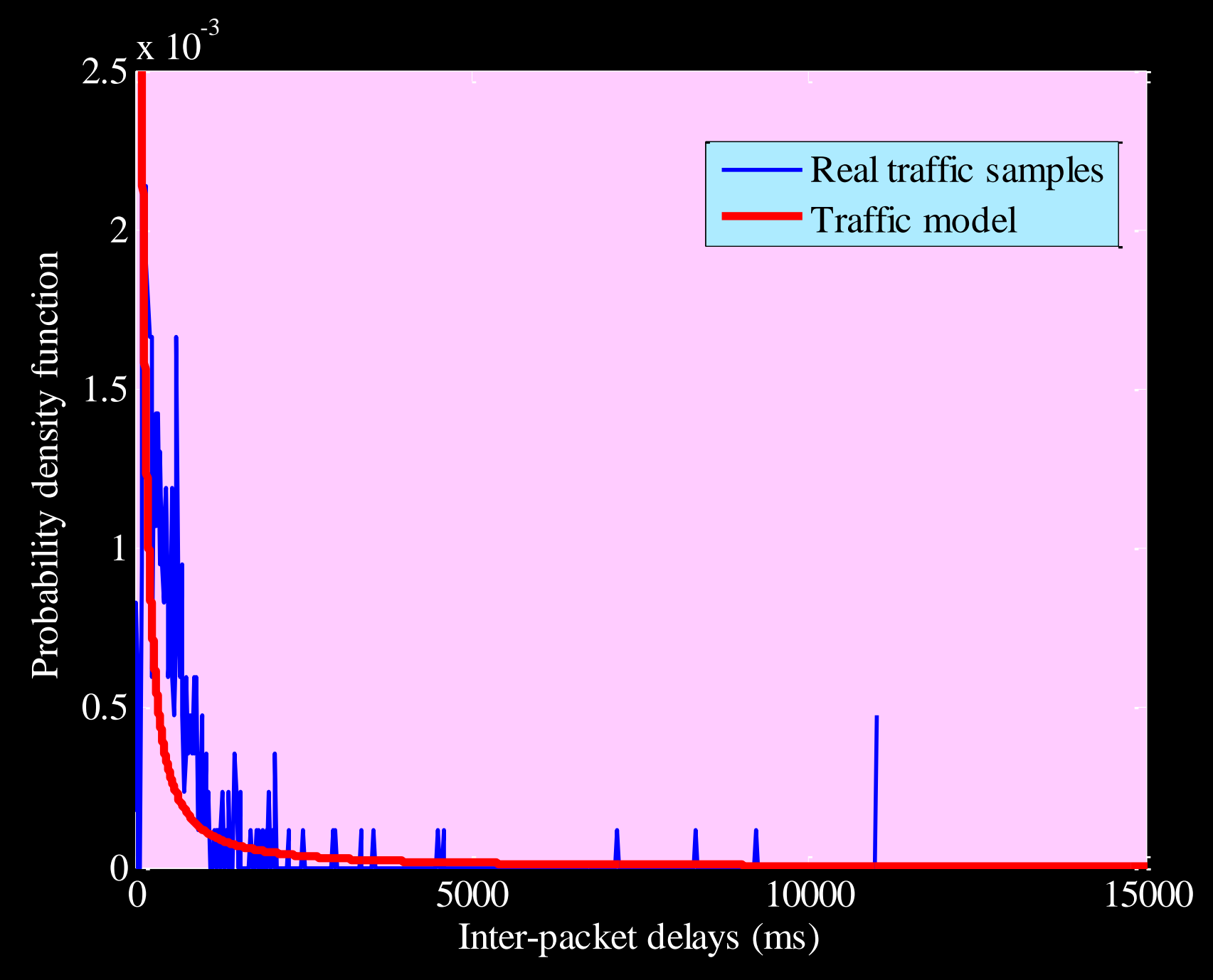

Traffic Modeling

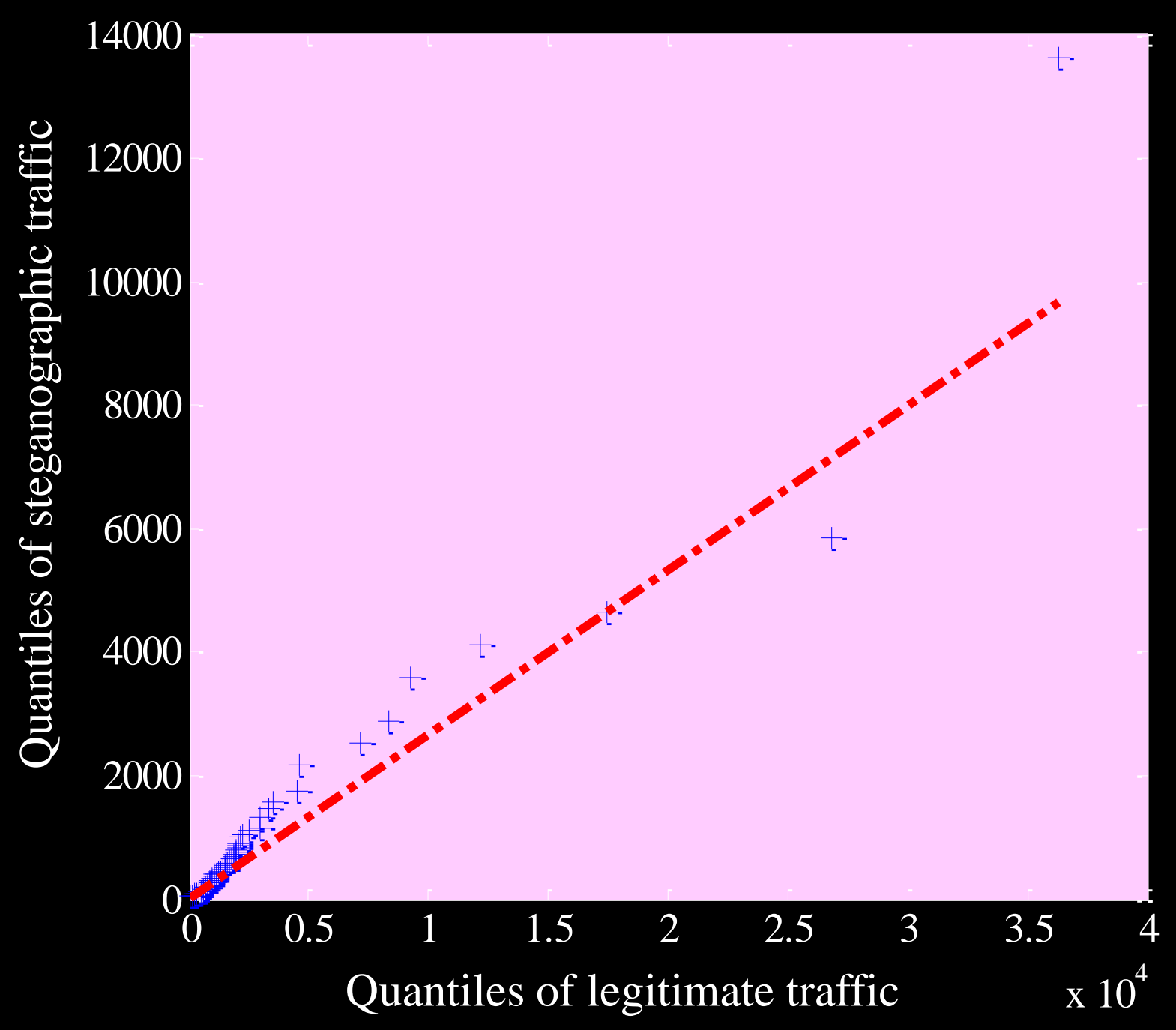

Q-Q plot 


\section{Robustness Evaluation}

Bit error rate $P_{e}$ for the experiments in the LAN

\begin{tabular}{|c|c|c|c|c|c|c|c|c|c|c|}
\hline \multirow{2}{*}{\multicolumn{2}{|c|}{ Encoding scheme }} & \multirow{2}{*}{ LAN } & \multicolumn{4}{|c|}{ Gaussian $\sigma^{2}\left(\mathrm{~ms}^{2}\right)$} & \multicolumn{4}{|c|}{ Uniform $\Delta^{2} / 12\left(\mathrm{~ms}^{2}\right)$} \\
\hline & & & 100 & 200 & 400 & 900 & 100 & 200 & 400 & 900 \\
\hline \multirow{3}{*}{$\gamma$ spreading } & 1 & 0 & 4.67 & 9.97 & 24.87 & 33.97 & 18.93 & 31.34 & 52.01 & 67.43 \\
\hline & 5 & 0 & 0 & 0.0003 & 0.23 & 1.27 & 0.20 & 1.13 & 6.33 & 20.37 \\
\hline & 10 & 0 & 0 & 0 & 0 & 3.63 & 0 & 0 & 0.60 & 4.33 \\
\hline
\end{tabular}

Bit error rate $P_{e}$ for the experiments in the WAN

\begin{tabular}{|c|c|c|c|c|c|c|c|c|c|c|}
\hline \multirow{2}{*}{\multicolumn{2}{|c|}{ Encoding scheme }} & \multirow{3}{*}{$\begin{array}{l}\text { WAN } \\
0.02\end{array}$} & \multicolumn{4}{|c|}{ Gaussian $\sigma^{2}\left(\mathrm{~ms}^{2}\right)$} & \multicolumn{4}{|c|}{ Uniform $\Delta^{2} / 12\left(\mathrm{~ms}^{2}\right)$} \\
\hline & & & 100 & 200 & 400 & 900 & 100 & 200 & 400 & 900 \\
\hline \multirow{3}{*}{$\gamma$ spreading } & 1 & & 6.01 & 10.22 & 26.93 & 34.98 & 20.10 & 33.23 & 55.89 & 69.87 \\
\hline & 5 & 0 & 0.0006 & 0.01 & 0.26 & 1.56 & 0.44 & 1.78 & 8.29 & 23.67 \\
\hline & 10 & 0.01 & 0 & 0 & 0.0003 & 4.01 & 0 & 0 & 1.23 & 5.64 \\
\hline
\end{tabular}




\section{Evaluation Tradeoff}

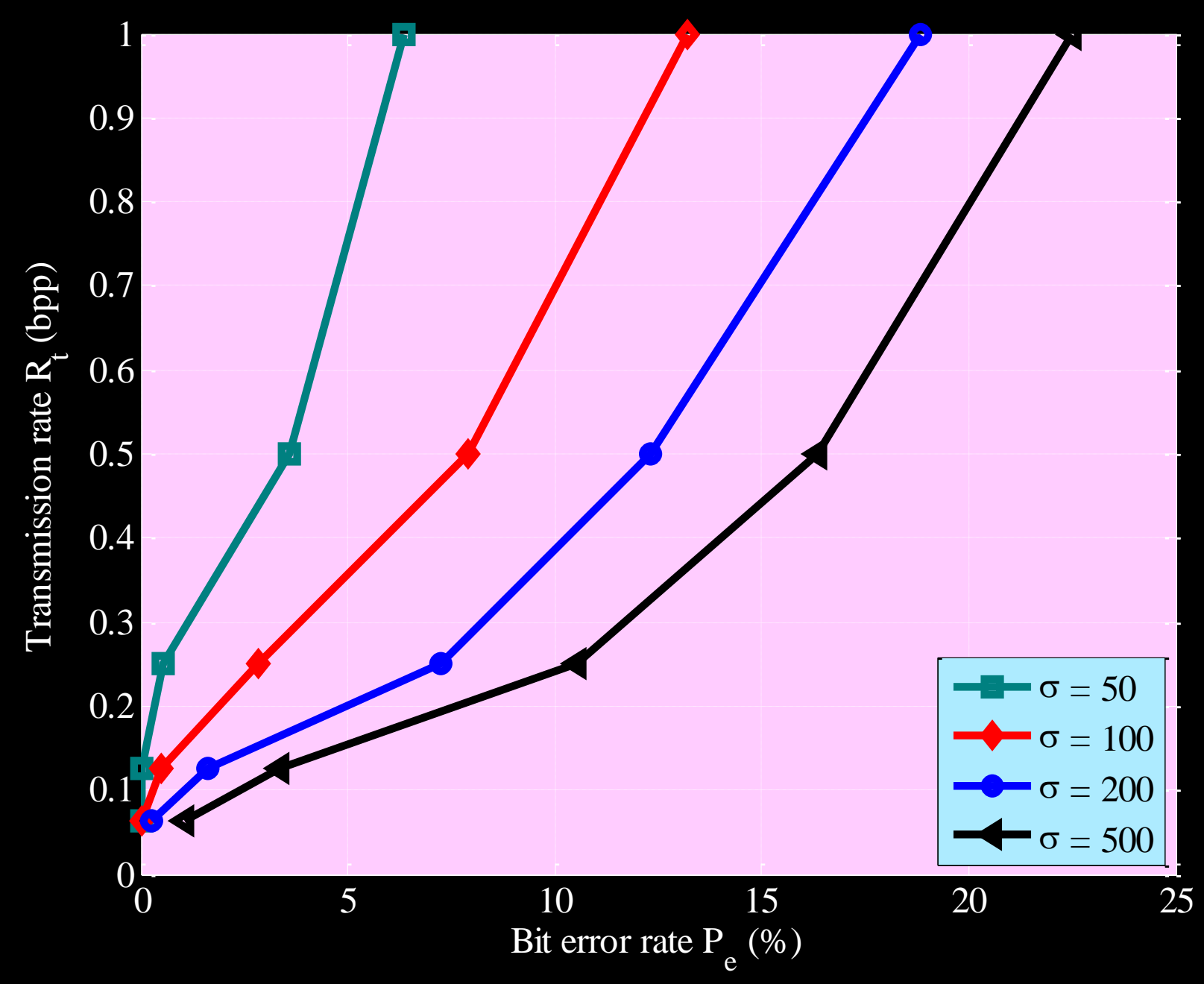

The performance trade-off between the transmission rate $R_{t}$ and bit error rate $P_{e}$ (under jammed uniform noise) . 


\section{Conclusion, Discussion, Future Work}

$\diamond$ We propose a method to modulate a steganographic timing channel on network traffic with independent and identically distributed (i.i.d.) inter-packet delays.

$\diamond$ It is both robust and provably undetectable and allows to balance

$\diamond$ Robustness against network noise

$\diamond$ Transmission rate

$\diamond$ We experimentally validate establishing steganographic channel using real Telnet traffic

Work in progress

$\diamond$ Extension of our approach for real applications such as video streaming or Voice over IP (VOIP) 